University of Nebraska - Lincoln

DigitalCommons@University of Nebraska - Lincoln

Mechanical \& Materials Engineering Faculty

Publications

Mechanical \& Materials Engineering,

Department of

$12-20-2019$

\title{
Generalized ultrasonic scattering model for arbitrary transducer configurations
}

Andrea P. Arguelles

Pennsylvania State University, arguelles@psu.edu

Joseph A. Turner

University of Nebraska-Lincoln, jaturner@unl.edu

Follow this and additional works at: https://digitalcommons.unl.edu/mechengfacpub

Part of the Mechanics of Materials Commons, Nanoscience and Nanotechnology Commons, Other Engineering Science and Materials Commons, and the Other Mechanical Engineering Commons

Arguelles, Andrea P. and Turner, Joseph A., "Generalized ultrasonic scattering model for arbitrary transducer configurations" (2019). Mechanical \& Materials Engineering Faculty Publications. 468. https://digitalcommons.unl.edu/mechengfacpub/468

This Article is brought to you for free and open access by the Mechanical \& Materials Engineering, Department of at DigitalCommons@University of Nebraska - Lincoln. It has been accepted for inclusion in Mechanical \& Materials Engineering Faculty Publications by an authorized administrator of DigitalCommons@University of Nebraska Lincoln. 


\title{
Generalized ultrasonic scattering model for arbitrary transducer configurations
}

\author{
Andrea P. Arguelles ${ }^{1, a)}$ and Joseph A. Turner ${ }^{2}$ \\ ${ }^{1}$ Department of Engineering Science and Mechanics, The Pennsylvania State University, University Park, \\ Pennsylvania 16802, USA \\ ${ }^{2}$ Department of Mechanical and Materials Engineering, University of Nebraska-Lincoln, Lincoln, \\ Nebraska 68588, USA
}

(Received 10 April 2019; revised 27 October 2019; accepted 12 November 2019; published online 20 December 2019)

\begin{abstract}
Ultrasonic scattering in polycrystalline media is directly tied to microstructural features. As a result, modeling efforts of scattering from microstructure have been abundant. The inclusion of beam modeling for the ultrasonic transducers greatly simplified the ability to perform quantitative, fully calibrated experiments. In this article, a theoretical scattering model is generalized to allow for arbitrary source and receiver configurations, while accounting for beam behavior through the total propagation path. This extension elucidates the importance and potential of out-of-plane scattering modes in the context of microstructure characterization. The scattering coefficient is explicitly written for the case of statistical isotropy and ellipsoidal grain elongation, with a direct path toward expansion for increased microstructural complexity. Materials with crystallites of any symmetry can be studied with the present model; the numerical results focus on aluminum, titanium, and iron. The amplitude of the scattering response is seen to vary across materials, and to have varying sensitivity to grain elongation and orientation depending on the transducer configuration selected. The model provides a pathway to experimental characterization of microstructure with optimized sensitivity to parameters of interest. (C) 2019 Acoustical Society of America. https://doi.org/10.1121/1.5139220
\end{abstract}

[MDV]

Pages: 4413-4424

\section{INTRODUCTION}

The diffuse field of ultrasonic waves can be characterized by scattering coefficients. Ultrasonic scattering coefficients are defined as the total energy scattered in a given direction. The scattering coefficients for metals with cubic crystallites were first derived by extending flaw scattering models ${ }^{1,2}$ and reciprocity relationships. ${ }^{3-5}$ Rose $^{6}$ used Auld's ${ }^{4}$ theory to derive backscatter coefficients directly related to the signal observed in an oscilloscope (backscatter refers to the signals scattered in the backward direction from the incident wave). Margetan et $a l^{7}$ obtained equivalent expressions using the reciprocity relations of Thompson and Gray. ${ }^{5}$ The models of Rose ${ }^{6}$ and Margetan et al. ${ }^{7}$ have been modified to account for more complex microstructures. Others considered titanium alloys with multiple phases and grain elongation. ${ }^{8-11}$ Han et al. ${ }^{12}$ included texture using Roe's orientation distribution function ${ }^{13}$ and $\mathrm{Li}$ and Thompson ${ }^{14}$ considered hexagonal crystallites. The models were later extended to include angular dependence of scattering coefficients within the material. ${ }^{15}$ Lobkis et al. ${ }^{16,17}$ included duplex microstructure and elongated grains. Sha ${ }^{18}$ included grain size distributions while $\mathrm{Li}$ and Rokhlin $^{22}$ accounted for macrotexture and grain elongation. Others extended to texture and lower crystal symmetries. ${ }^{19-23}$

Ghoshal et al. ${ }^{24}$ established a mathematical formalism within a multiple scattering framework that included transducer modeling. The scattered response was given as a convolution of the displacement fields' Wigner transforms with an intensity operator defined by the Bethe-Salpeter equation. ${ }^{24}$ This form

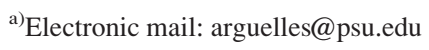

of the intensity operator (associated with the scattering coefficient) allows for a full expansion to multiple scattering with the authors providing a solution for the singly-scattered response (SSR). ${ }^{24}$ This formulation of the scattering response rigorously accounts for the transducer beam energy as it passes through a coupling fluid and into the solid, facilitating correlation with experimental results. Some simplifying assumptions have been made to obtain closed-form solutions of the SSR expression. Multiple authors have explored the applicability of this solution for extraction of microstructural features of polycrystalline media. ${ }^{25-28}$ Ghoshal and Turner ${ }^{25}$ provided a solution for the longitudinal backscatter response through a curved interface. $\mathrm{Hu}$ et al. extended the model to in-plane mode-converted ${ }^{26}$ and shear-to-shear ${ }^{27}$ scattering, which are advantageous for thinner samples and detection of defects in the transverse direction. Arguelles et al. ${ }^{28}$ then considered the mode-converted scattering model in the presence of grain elongation. In all these studies, ${ }^{25-28}$ the authors were successful at inverting ultrasonic data to obtain characteristic grain sizes.

In this article, Ghoshal and Turner's model ${ }^{25}$ is extended to arbitrary wave propagation directions for the source and receiver which can have longitudinal or shear incident and scattered waves. A measurement model is included which accounts for transmission and reflection at the interfaces as well as full beam modeling for all configurations of source and receiver. This result represents advancement over previous arbitrary scattering direction models, ${ }^{15}$ which accounted only for scattering contributions within the material. First, a review of the SSR model given by Ghoshal et al. ${ }^{24}$ is given in Sec. II. In Sec. II A, the transducer fields are written in terms of independent coordinate systems, which are then related to 
the fixed coordinates used for the spatial integration of the scattered energy. As presented in Sec. II B, the model given herein is applicable to microstructures for which the spatial component of the scattering coefficient can be separated from the tensorial component; the tensorial term represents the variations in the elastic constants of the medium. This model is then reduced to the case of macroscopic isotropy, for which the crystallites are assumed to be randomly oriented with a closed-form expression given in Sec. IIC. In Sec. III, the effect of grain elongation on the scattering response is studied in detail. Numerical results concentrate on the out-of-plane shear-to-shear scattering, which is the primary extension to other closed-form solutions of Ghoshal and Turner's model. ${ }^{25}$ This generalized model will allow components with increased microstructural complexity to be characterized.

\section{SINGLE SCATTERING RESPONSE MODEL}

Ghoshal et al., ${ }^{24}$ under the assumption of single scattering, derived an analytical expression for the spatial variance of signals captured at various positions on a sample. This time-dependent variance, SSR, is given by

$$
\begin{aligned}
\Phi(t)= & \gamma_{S} \gamma_{R} \int \frac{d \omega}{(2 \pi)^{4}} d \mathbf{k} d \mathbf{k}^{\prime} d \mathbf{x} d t^{\prime} W_{\beta j}^{R}\left(\mathbf{x}, t-t^{\prime}, \mathbf{k}^{\prime}, \omega\right) \\
& \times{ }_{\mathbf{k}^{\prime} j}^{\mathbf{k}^{\prime} \beta} K_{k \mathbf{k}}^{\gamma \mathbf{k}} W_{\gamma k}^{S}\left(\mathbf{x}, t^{\prime}, \mathbf{k}, \omega\right),
\end{aligned}
$$

where $\gamma_{S}$ and $\gamma_{R}$ relate the displacement fields to the transducer voltages and are obtained through calibration experiments. This general expression allows for arbitrary mode types of the incident and scattered fields, given by $\mathbf{k}$ and $\mathbf{k}^{\prime}$, respectively. Equation (1) is an integral of the inner product of $\mathbf{W}^{S}$ and $\mathbf{W}^{R}$ with the intensity operator $\mathbf{K}$ associated with the heterogeneous medium, and includes a temporal convolution of $\mathbf{W}^{S}$ and $\mathbf{W}^{R}$. $\mathbf{W}^{S}$ and $\mathbf{W}^{R}$ are the transducer energy distributions, quantified by the four-fold Wigner transform of the displacement fields created by the source and receiving transducers, respectively. More specifically, $\mathbf{W}$ represents the signal in the space-time $(\mathbf{x}, t)$ and wave vector-frequency $(\mathbf{k}, \omega)$ domains simultaneously. Section II A provides $\mathbf{W}$ for a piston transducer placed relative to a sample in an arbitrary orientation and Sec. II B describes $\mathbf{K}$ for different microstructures.

\section{A. Wigner transform of piston transducer at arbitrary angles}

The four-fold Wigner transform can be written as

$$
\begin{aligned}
W_{\beta j}(\mathbf{x}, t, \mathbf{k}, \omega)= & \int\left\langle\Psi_{\beta}(\mathbf{x}+\xi / 2, t+\tau / 2)\right\rangle \\
& \times\left\langle\Psi_{j}(\mathbf{x}-\xi / 2, t-\tau / 2)\right\rangle \\
& \times \exp \{i \mathbf{k} \cdot \xi+i \omega \tau\} d^{3} \xi d \tau,
\end{aligned}
$$

where $\Psi$ is the displacement field and the angular brackets \langle\rangle denote the ensemble average. The Wigner transform was first calculated for a piston transducer by Ghoshal et al., ${ }^{24}$ and explicitly written for longitudinal propagation at normal incidence in Ghoshal and Turner ${ }^{25}$ and for shear oblique incidence in $\mathrm{Hu}$ et $a l .^{26}$ Here, the expression for $\mathbf{W}$ is generalized for arbitrary wave modes and propagation directions as depicted in Fig. 1. The Wigner transform for the source becomes

$$
\begin{aligned}
& W_{\gamma k}^{S}(\mathbf{x}, t, \mathbf{k}, \omega) \\
& =T_{f \chi}^{2} A_{0 S}^{2}(2 \pi)^{3} \sqrt{2 \pi} \sigma_{S} \frac{w_{0 S}^{2}}{w_{1}\left(Z_{S}\right) w_{2}\left(Z_{S}\right)} \\
& \quad \times \exp \left[-\frac{2 X_{S}^{2}}{w_{1}^{2}\left(Z_{S}\right)}-\frac{2 Y_{S}^{2}}{w_{2}^{2}\left(Z_{S}\right)}\right] \\
& \quad \times \exp \left[-\frac{2 Z_{S}\left(Z_{S}-2 t c_{\chi}\right)}{\sigma_{S}^{2} c_{\chi}^{2}}-2 \alpha_{\chi} Z_{S}-\frac{1}{2} \sigma_{S}^{2}\left(\omega-\omega_{0 S}\right)^{2}\right] \\
& \quad \times \exp \left[-2\left(\frac{t}{\sigma_{S}}\right)^{2}\right] \delta^{3}\left(\mathbf{k}-\mathbf{k}_{0}\right) \hat{e}_{\gamma} \hat{e}_{k}\left(\hat{\mathbf{k}} \cdot \hat{\mathbf{n}}_{S}\right)^{2}
\end{aligned}
$$

where a local coordinate system $\mathbf{X}_{\mathbf{S}}$ is used, as shown in Fig. 2. The Wigner transform for the receiver $\mathbf{W}^{R}$ can be cast in a similar form using a local coordinate system $\mathbf{X}_{\mathbf{R}}$ and distinct wave properties for the scattered wave, so that

$$
\begin{aligned}
& W_{\beta j}^{R}\left(\mathbf{x}, t, \mathbf{k}^{\prime}, \omega\right) \\
& =T_{\chi^{\prime} f}^{2} A_{0 R}^{2}(2 \pi)^{4} \frac{w_{0 R}^{2}}{w_{1}\left(Z_{R}\right) w_{2}\left(Z_{R}\right)} \\
& \times \exp \left[-\frac{2 X_{R}^{2}}{w_{1}^{2}\left(Z_{R}\right)}-\frac{2 Y_{R}^{2}}{w_{2}^{2}\left(Z_{R}\right)}\right] \\
& \times \exp \left[-\frac{2 Z_{R}\left(Z_{R}-2 t c_{\chi^{\prime}}\right)}{\sigma_{R}^{2} c_{\chi^{\prime}}^{2}}-2 \alpha_{\chi^{\prime}} Z_{R}\right] \delta\left(\omega-\omega_{0 R}\right) \\
& \times \exp \left[-2\left(\frac{t}{\sigma_{R}}\right)^{2}\right] \delta^{3}\left(\mathbf{k}^{\prime}-\mathbf{k}_{0}^{\prime}\right) \hat{e}_{\beta}^{\prime} \hat{e}_{j}^{\prime}\left(\hat{\mathbf{k}}^{\prime} \cdot \hat{\mathbf{n}}_{R}\right)^{2} .
\end{aligned}
$$

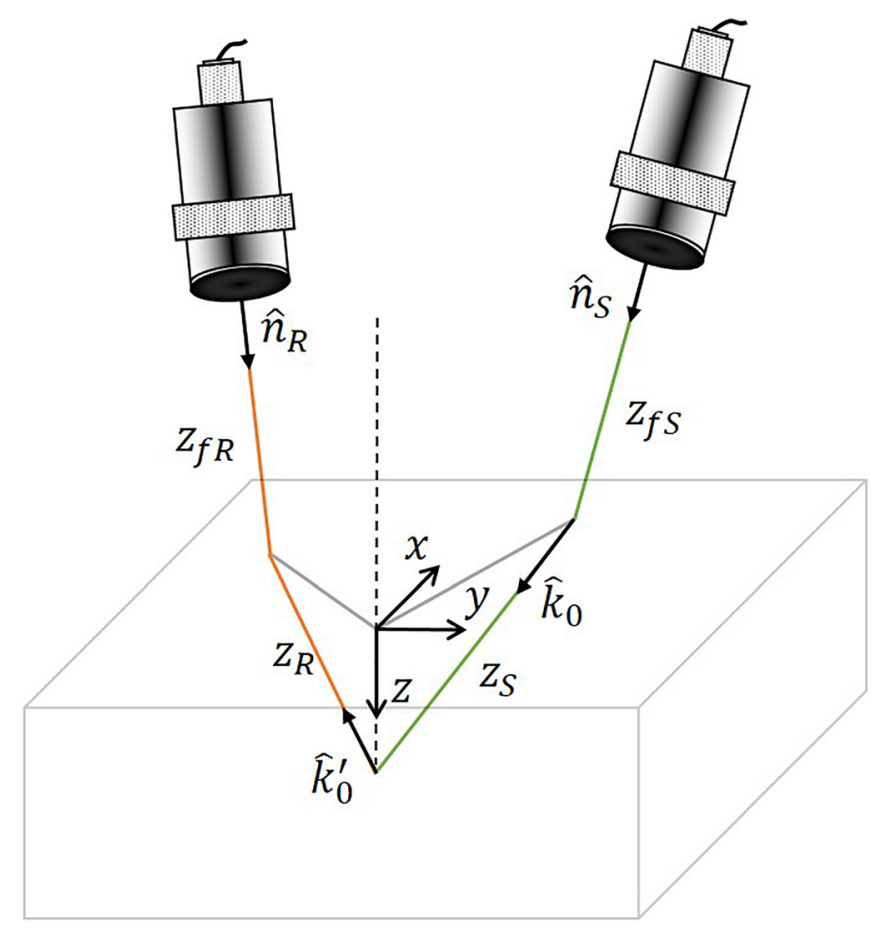

FIG. 1. (Color online) Schematic diagram of a pitch-catch transducer configuration with non-coplanar source and receiver. 

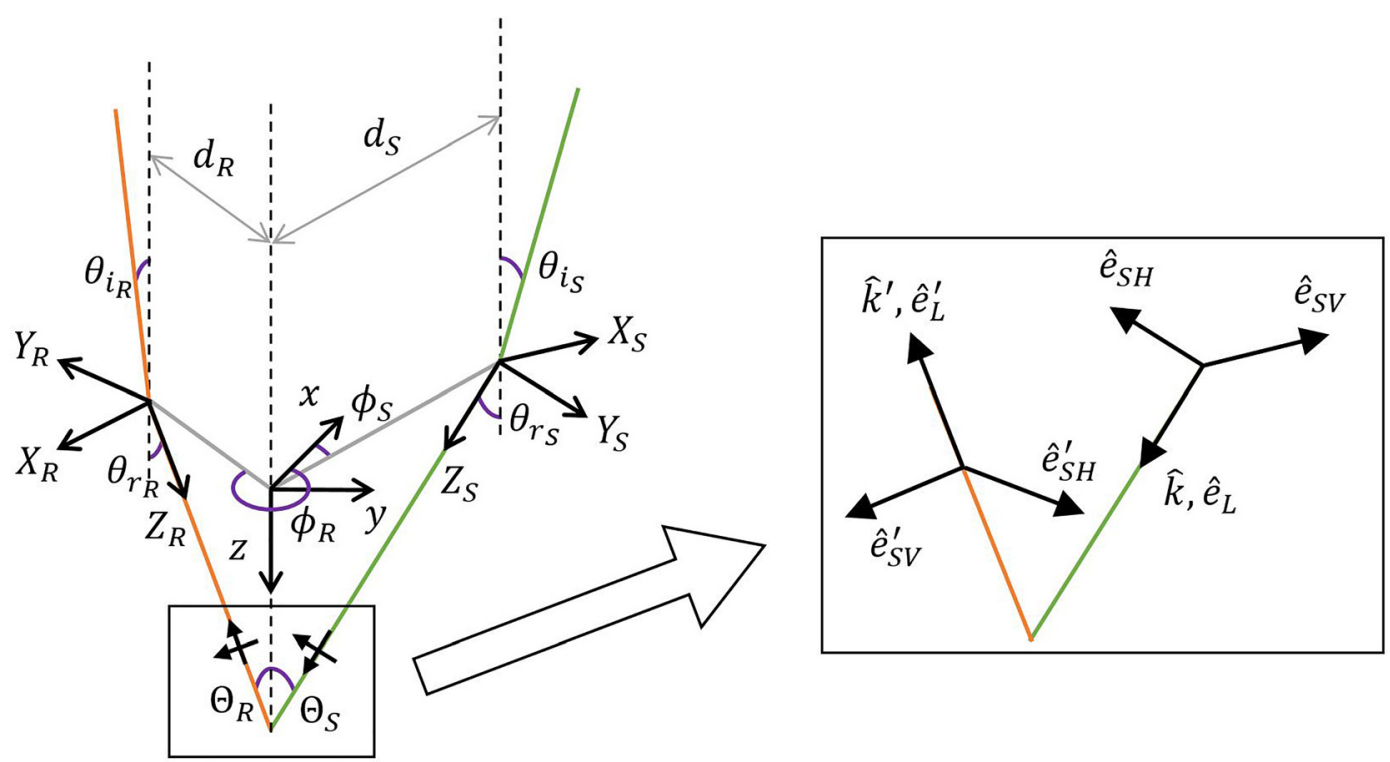

FIG. 2. (Color online) Coordinate transformation diagram for non-coplanar source and receiver with respect to a fixed global coordinate system.

In order to simplify later integrations, the receiving transducer is assumed to have a single frequency $\omega_{0 R}$ rather than a Gaussian distribution of frequencies. ${ }^{24-26}$ In Eqs. (3) and (4), the variables are designated as follows:

$\chi, \chi^{\prime}$-wave type for incident and scattered waves, $A_{0}$-amplitude correction due to propagation in fluid, ${ }^{29}$

$T_{f \chi}, T_{\chi^{\prime} f}$-transmission coefficients, fluid to sample and sample to fluid,

$c_{L}, c_{T}$-longitudinal and shear wave speeds,

$\alpha_{L}, \alpha_{T}$-longitudinal and shear wave attenuations,

$\sigma_{S}, \sigma_{R}$-pulse width for source and receiving transducers,

$\omega_{0 S}, \omega_{0 R}-$ center frequencies for source and receiving transducers,

$\mathbf{k}, \mathbf{k}^{\prime}$-propagation direction vectors for the incident and scattered waves,

$\hat{\mathbf{e}}, \hat{\mathbf{e}}^{\prime}$-displacement directions for the incident and scattered waves,

$\hat{\mathbf{n}}_{S}, \hat{\mathbf{n}}_{R}$-unit vectors normal to the surface of the source and receiving transducers.

Assuming a single Gaussian beam in space for the piston transducers, ${ }^{24}$

$w_{0}$-initial beam width,

$w_{1}, w_{2}$-widths of the Gaussian profile along the propagation axis $z,{ }^{30}$

expressions for which are given in Appendix A. This approximation is applicable to both flat and focused transducers. Because the spatial integration in Eq. (1) is written in the global coordinate system $\mathbf{x}$, a coordinate transformation from $\mathbf{X}_{\xi}$ to $\mathbf{x}$ is necessary. As depicted in Fig. 2, a simple geometric relation given by the refraction angle $\Theta_{\xi}$ and rotation angle $\phi_{\xi}$ can be written as

$$
\begin{aligned}
X_{\xi}= & x \cos \phi_{\xi} \cos \Theta_{\xi}+y \sin \phi_{\xi} \cos \Theta_{\xi} \\
& +z \sin \Theta_{\xi}-d_{\xi} \cos \Theta_{\xi}, \\
Y_{\xi}= & -x \sin \phi_{\xi}+y \cos \phi_{\xi}, \\
Z_{\xi}= & -x \cos \phi_{\xi} \sin \Theta_{\xi}-y \sin \phi_{\xi} \sin \Theta_{\xi} \\
& +z \cos \Theta_{\xi}+d_{\xi} \sin \Theta_{\xi} .
\end{aligned}
$$

The subscript $\xi$ is used to differentiate the transducers ( $\xi=S, R$, source or receiver, respectively).

\section{B. Intensity operator, $\mathrm{K}$}

Having characterized the transducer energy distributions $\mathbf{W}$, the next step is to define the intensity operator $\mathbf{K}$ in Eq. (1). $\mathbf{K}$ is an eighth-rank tensor that quantifies the scattering within the material, written here as

$$
\begin{aligned}
& \mathbf{k}^{\prime} \beta \\
& \mathbf{k}^{\prime} j \\
& K_{k \mathbf{k}}^{\gamma \mathbf{k}} \approx \tilde{\eta}\left(\mathbf{k}^{\prime}-\mathbf{k}\right) \mathbf{k}_{\alpha}^{\prime} \mathbf{k}_{\delta} \mathbf{k}_{l}^{\prime} \mathbf{k}_{m} \Xi_{l m j k}^{\alpha \delta \beta \gamma} \\
&=k^{\prime 2} k^{2} \tilde{\eta}\left(k^{\prime} \hat{\mathbf{k}}^{\prime}-k \hat{\mathbf{k}}\right) \hat{k}_{\alpha}^{\prime} \hat{k}_{\delta} \hat{k}_{l}^{\prime} \hat{k}_{m} \Xi_{l m j k}^{\alpha \delta \beta \gamma},
\end{aligned}
$$

assuming the spatial and tensorial components are independent of each other. ${ }^{31}$ The notation ${ }_{\mathbf{k}^{\prime} j}^{\mathbf{k}^{\prime} \beta} K_{k \mathbf{k}}^{\gamma \mathbf{k}}$ denotes a wave propagating in the $\hat{\mathbf{k}}$ direction that scatters into the $\hat{\mathbf{k}}^{\prime}$ direction. The function $\tilde{\eta}\left(\mathbf{k}^{\prime}-\mathbf{k}\right)$ is the spatial Fourier transform of the two-point probability function giving the likelihood that two randomly chosen positions will lie within a given grain. Hence, $\tilde{\eta}$ is a function of the difference between the incident and scattered wave vectors due to the implicit assumption of statistical homogeneity. $\Xi_{l m j k}^{\alpha \delta \beta \gamma}$ is the elastic modulus covariance given by $\Xi_{l m j k}^{\alpha \delta \beta \gamma}=\left\langle C_{l m j k} C_{\alpha \delta \beta \gamma}\right\rangle-\left\langle C_{l m j k}\right\rangle\left\langle C_{\alpha \delta \beta \gamma}\right\rangle$, which represents the second-order statistics of the spatial distribution of crystal orientations responsible for the scattering. $\tilde{\eta}\left(\mathbf{k}^{\prime}-\mathbf{k}\right)$ is described in more detail for elongated grains in Appendix B.

\section{Generalized model}

Now Eqs. (3), (4), and (6) can be substituted into Eq. (1). Generally, transducers used for these experiments have matched frequencies and pulse widths; hence, we can assume $\sigma_{S}=\sigma_{R}=\sigma$ and $\omega_{0 S}=\omega_{0 R}=\omega_{0}$ to obtain 


$$
\begin{aligned}
\Phi(t)= & \gamma_{S} \gamma_{R}\left(T_{f \chi} T_{\chi^{\prime} f}\right)^{2}\left(A_{0 R} A_{0 S}\right)^{2}(2 \pi)^{3} \sqrt{2 \pi} \sigma \int \frac{w_{0 R}^{2} w_{0 S}^{2}}{w_{1}\left(Z_{R}\right) w_{2}\left(Z_{R}\right) w_{1}\left(Z_{S}\right) w_{2}\left(Z_{S}\right)} \\
& \times \exp \left[-\frac{2 X_{R}^{2}}{w_{1}^{2}\left(Z_{R}\right)}-\frac{2 Y_{R}^{2}}{w_{2}^{2}\left(Z_{R}\right)}-\frac{2 Z_{R}\left(Z_{R}-2\left(t-t^{\prime}\right) c_{\chi^{\prime}}\right)}{\sigma^{2} c_{\chi^{\prime}}^{2}}-2\left(\frac{t-t^{\prime}}{\sigma}\right)^{2}\right] \\
& \times \exp \left[-2 \alpha_{\chi^{\prime}} Z_{R}-\frac{1}{2} \sigma^{2}\left(\omega-\omega_{0}\right)^{2}\right] \delta^{3}\left(\mathbf{k}^{\prime}-\mathbf{k}_{0}^{\prime}\right) \hat{e}_{\beta}^{\prime} \hat{e}_{j}^{\prime}\left(\hat{\mathbf{k}}^{\prime} \cdot \hat{\mathbf{n}}_{R}\right)^{2} \times k^{\prime 2} k^{2} \tilde{\eta}\left(k^{\prime} \hat{\mathbf{k}}^{\prime}-k \hat{\mathbf{k}}\right) \hat{k}_{\alpha}^{\prime} \hat{k}_{\delta} \hat{k}_{l}^{\prime} \hat{k}_{m} \Xi_{l m j k}^{\alpha \delta \beta \gamma} \\
& \times \exp \left[-\frac{2 X_{S}^{2}}{w_{1}^{2}\left(Z_{S}\right)}-\frac{2 Y_{S}^{2}}{w_{2}^{2}\left(Z_{S}\right)}-\frac{2 Z_{S}\left(Z_{S}-2 t^{\prime} c_{\chi}\right)}{\sigma^{2} c_{\chi}^{2}}-2\left(\frac{t^{\prime}}{\sigma}\right)^{2}-2 \alpha_{\chi} Z_{S}\right] \delta\left(\omega-\omega_{0}\right) \delta^{3}\left(\mathbf{k}-\mathbf{k}_{0}\right) \\
& \times \hat{e}_{\gamma} \hat{e}_{k}\left(\hat{\mathbf{k}} \cdot \hat{\mathbf{n}}_{S}\right)^{2} d \omega d \mathbf{k} d \mathbf{k}^{\prime} d x d y d z d t^{\prime} .
\end{aligned}
$$

Performing the integrations over $t^{\prime}, \mathbf{k}, \mathbf{k}^{\prime}$, and $\omega$, gives

$$
\begin{aligned}
\Phi(t)= & \gamma_{S} \gamma_{R}\left(T_{f \chi} T_{\chi^{\prime} f}\right)^{2}\left(A_{0 R} A_{0 S}\right)^{2}(2 \pi)^{4} \frac{\omega_{0}^{4}}{c_{\chi}^{2} c_{\chi^{\prime}}^{2}} \sigma^{2} \frac{\sqrt{2}}{4} \times \int d x d y d z \frac{w_{0 R}^{2} w_{0 S}^{2}}{w_{1}\left(Z_{R}\right) w_{2}\left(Z_{R}\right) w_{1}\left(Z_{S}\right) w_{2}\left(Z_{S}\right)} \exp \left[-\frac{2 X_{R}^{2}}{w_{1}^{2}\left(Z_{R}\right)}-\frac{2 X_{S}^{2}}{w_{1}^{2}\left(Z_{S}\right)}\right] \\
& \times \exp \left[-\frac{2 Y_{R}^{2}}{w_{2}^{2}\left(Z_{R}\right)}-\frac{2 Y_{S}^{2}}{w_{2}^{2}\left(Z_{S}\right)}-2 \alpha_{\chi^{\prime}} Z_{R}-2 \alpha_{\chi} Z_{S}-\frac{2 Z_{R}\left(Z_{R}-2 t c_{\chi^{\prime}}\right)}{\sigma^{2} c_{\chi^{\prime}}^{2}}-\frac{2 Z_{S}^{2}}{\sigma^{2} c_{\chi}^{2}}-2\left(\frac{t}{\sigma}\right)^{2}+\frac{1}{\sigma^{2}}\left(\frac{Z_{S}}{c_{\chi}}-\frac{Z_{R}}{c_{\chi^{\prime}}}+t\right)^{2}\right] \\
& \times \tilde{\eta}\left(k^{\prime} \hat{\mathbf{k}}_{0}^{\prime}-k \hat{\mathbf{k}}_{0}\right) \times \hat{k}_{\alpha}^{\prime} \hat{k}_{\delta} \hat{k}_{l}^{\prime} \hat{k}_{m} \Xi_{l m j k}^{\alpha \delta \beta \gamma} \hat{e}_{\gamma} \hat{e}_{k}\left(\hat{\mathbf{k}}_{0} \cdot \hat{\mathbf{n}}_{S}\right)^{2} \hat{e}_{\beta}^{\prime} \hat{e}_{j}^{\prime}\left(\hat{\mathbf{k}}_{0}^{\prime} \cdot \hat{\mathbf{n}}_{R}\right)^{2} .
\end{aligned}
$$

The coefficients $\gamma_{\xi}$ and $A_{0 \xi}$ are given by

$$
\begin{aligned}
& \gamma_{S}=V_{\max }^{S} \frac{2 \rho_{f} k_{f}^{2} \sigma c_{f}^{2}}{R_{f f} D_{S}\left(\omega_{0}\right)} \sqrt{\frac{2}{\pi}} \frac{w\left(z_{F S}\right)}{w_{0 S}^{2}}\left(\pi w_{0 S}^{2}\right)^{2} \exp \left(2 \alpha_{f} z_{F S}\right) \\
& \gamma_{R}=V_{\max }^{R} \frac{2 \rho_{f} k_{f}^{2} \sigma c_{f}^{2}}{R_{f f} D_{R}\left(\omega_{0}\right)} \sqrt{\frac{2}{\pi}} \frac{w\left(z_{F R}\right)}{w_{0 R}^{2}}\left(\pi w_{0 R}^{2}\right)^{2} \exp \left(2 \alpha_{f} z_{F R}\right) \\
& A_{0 S}=-\frac{\exp \left(-\alpha_{f} z_{f S}\right)}{4 \pi w_{0 S}^{2} \rho_{f} c_{f}^{2} \sigma k_{f} \sqrt{\pi}} \\
& A_{0 R}=-\frac{\exp \left(-\alpha_{f} z_{f R}\right)}{4 \pi w_{0 R}^{2} \rho_{f} c_{f}^{2} \sigma k_{f} \sqrt{\pi}}
\end{aligned}
$$

where $V_{\max }^{\xi}$ are the maximum signal voltages for the individual transducers (assuming reflection off a planar surface at normal incidence). The water paths used during the calibration procedure are given by $z_{F S}$ and $z_{F R}$, which are the focal lengths of the corresponding transducers. Details of the calibration procedure can be found elsewhere. ${ }^{25}$ The reflection coefficient $R_{f f}=\left(\rho c_{L}-\rho_{f} c_{f}\right) /\left(\rho c_{L}+\rho_{f} c_{f}\right)$ and the diffraction constant $D_{\xi}\left(\omega_{0}\right)=\left|1-e^{-\left(2 \pi i / s_{\xi}\right)}\left[J_{0}\left(2 \pi / s_{\xi}\right)+i J_{1}\left(2 \pi / s_{\xi}\right)\right]\right|^{32}$ where $s_{\xi}$ $=4 \pi c_{f} z_{F \xi} / \omega_{0} w_{0 \xi}^{2}$. Based on the unit vector relationships depicted in Figs. 1 and 2, the inner products are given by $\hat{\mathbf{k}}_{0} \cdot \hat{\mathbf{n}}_{S}=\cos \left(\Theta_{S}-\theta_{i_{S}}\right)$ and $\hat{\mathbf{k}}_{0}^{\prime} \cdot \hat{\mathbf{n}}_{R}=-\cos \left(\Theta_{R}-\theta_{i_{R}}\right)$. Finally, Eq. (8) becomes

$$
\begin{aligned}
\Phi(t)= & \Phi_{0} B \int d x d y d z \frac{1}{w_{1}\left(Z_{R}\right) w_{2}\left(Z_{R}\right) w_{1}\left(Z_{S}\right) w_{2}\left(Z_{S}\right)} \exp \left[-\frac{2 X_{R}^{2}}{w_{1}^{2}\left(Z_{R}\right)}-\frac{2 X_{S}^{2}}{w_{1}^{2}\left(Z_{S}\right)}\right] \\
& \times \exp \left[-\frac{2 Y_{R}^{2}}{w_{2}^{2}\left(Z_{R}\right)}-\frac{2 Y_{S}^{2}}{w_{2}^{2}\left(Z_{S}\right)}-\frac{2 Z_{R}^{2}}{\sigma^{2} c_{\chi^{\prime}}^{2}}-\frac{2 Z_{S}^{2}}{\sigma^{2} c_{\chi}^{2}}+\frac{1}{\sigma^{2}}\left(\frac{Z_{S}}{c_{\chi}}-\frac{Z_{R}}{c_{\chi^{\prime}}}\right)^{2}\right] \\
& \times \exp \left[\frac{2}{\sigma^{2}}\left(\frac{Z_{S}}{c_{\chi}}+\frac{Z_{R}}{c_{\chi^{\prime}}}\right) t-\left(\frac{t}{\sigma}\right)^{2}-2 \alpha_{\chi^{\prime}} Z_{R}-2 \alpha_{\chi} Z_{S}\right]
\end{aligned}
$$

where

$$
\begin{aligned}
\Phi_{0}= & \frac{\pi \sqrt{2}}{8} V_{\max }^{S} V_{\max }^{R}\left(\frac{T_{f \chi} T_{\chi^{\prime} f}}{R_{f f}}\right)^{2}\left(\frac{1}{\rho_{f} c_{f}^{2}}\right)^{2} \frac{w\left(z_{F S}\right) w\left(z_{F R}\right)}{D_{S}\left(\omega_{0}\right) D_{R}\left(\omega_{0}\right)} \\
& \times \cos ^{2}\left(\Theta_{S}-\theta_{i_{S}}\right) \times \cos ^{2}\left(\Theta_{R}-\theta_{i_{R}}\right) \\
& \times \exp \left[2 \alpha_{f}\left(z_{F S}-z_{f S}\right)+2 \alpha_{f}\left(z_{F R}-z_{f R}\right)\right]
\end{aligned}
$$

the previously stated assumptions of statistical homogeneity and isotropy of the second-order statistics of the polycrystal, the scattering coefficient may be written as

$$
B=\left(\frac{\omega_{0}^{2}}{c_{\chi} c_{\chi^{\prime}}}\right)^{2} \tilde{\eta}\left(k^{\prime} \hat{\mathbf{k}}_{0}^{\prime}-k \hat{\mathbf{k}}_{0}\right) \hat{k}_{\alpha}^{\prime} \hat{k}_{\delta} \hat{e}_{\beta}^{\prime} \hat{e}_{\gamma} \hat{k}_{l}^{\prime} \hat{k}_{m} \hat{e}_{j}^{\prime} \hat{e}_{k} \Xi_{l m j k}^{\alpha \delta \beta \gamma} .
$$

$\Phi_{0}$ is a factor related to experiment calibration and $B$ is the scattering coefficient. The expression given by Eq. (10) is applicable to materials with various microstructures. With

Details of the inner product $\hat{k}_{\alpha}^{\prime} \hat{k}_{\delta} \hat{e}_{\beta}^{\prime} \hat{e}_{\gamma} \hat{k}_{l}^{\prime} \hat{k}_{m} \hat{e}_{j}^{\prime} \hat{e}_{k} \Xi_{l m j k}^{\alpha \delta \beta \gamma}$ in the scattering coefficient are given in Appendix $\mathrm{C}$ for various 
incident-to-scattered wave combinations and arbitrary single crystal symmetry. Equation (10) is the primary result of this paper. This model allows for arbitrary transducer configurations, which expands the capabilities of scattering measurements for microstructure characterization. Although increasing the complexity of the model, a clear understanding of the scattering response for arbitrary transducer angles can improve current techniques used to determine grain morphology. Given the large number of variables in the generalized model, only select examples of transducer configurations are considered in Sec. III.

\section{NUMERICAL RESULTS}

The model given by Eq. (10) has a large number of possible configurations. In this section, a subset of those is numerically evaluated for select materials. The results presented consider only shear-to-shear scattering where $\theta_{i_{S}}$ and $\theta_{i_{R}}$ are greater than the first critical angle, $\theta_{I}$, for each material. Both in-plane $\left(\phi_{S}=\phi_{R}\right)$ and out-of-plane $\left(\phi_{S} \neq \phi_{R}\right)$ scattering configurations are considered. The foci of the transducers are chosen to overlap by adjusting the water and material paths for the source $\left(z_{f S}\right.$ and $\left.z_{S}\right)$ and the receiver $\left(z_{f R}\right.$ and $\left.z_{R}\right)$. For all examples, the frequency, focal length, and element radius of the source and receiver are fixed $(f=8 \mathrm{MHz}, F=50.8 \mathrm{~mm}$, and $a=4.76 \mathrm{~mm}$ ). The beam cross-section is assumed circular in all cases, which is deemed acceptable for the small transducer radius. Parameters of interest for selected materials are given in Table I. The fluid is selected to be ethylene glycol, with $c_{f}=1660 \mathrm{~m} / \mathrm{s}, \rho_{f}=1115 \mathrm{~kg} / \mathrm{m}^{3}$, and $\alpha_{f}=0.12 \times f^{2}$ $\mathrm{Np} / \mathrm{m}$, where $f$ is in hertz. ${ }^{33}$

Consider the following grain dimensions: $a_{\mathrm{x}}=15 \mu \mathrm{m}$, $a_{\mathrm{y}}=60 \mu \mathrm{m}, a_{\mathrm{z}}=30 \mu \mathrm{m}$. Figure 3 is given to illustrate the polar angles of the grain orientation, denoted by $\psi_{g}$, with respect to the transducer configuration, denoted by angles $\phi_{S}$ and $\phi_{R}$ for the source and receiver, respectively. This twodimensional illustration corresponds with the $x-y$ plane in Fig. 1. For a grain that is elongated along its $y$-direction, $\psi_{g}=0$ and $\phi_{S / R}=0$ correspond with wave propagation perpendicular to the direction of elongation. To simplify later examples, primary grain elongation will be set along the grain's $y$-direction, and the source transducer will remain fixed at $\phi_{S}=0^{\circ}$.

\section{A. Time-dependent scattering response}

Consider the case for which the incident and scattering angles are equal, $\Theta_{S}=\Theta_{R}=\Theta$; consequently, the material paths for the source and receiver are equal, $z_{S}=z_{R}$. For $a_{\mathrm{x}}=15 \mu \mathrm{m}, a_{\mathrm{y}}=60 \mu \mathrm{m}, a_{\mathrm{z}}=30 \mu \mathrm{m}$, and $\psi_{\mathrm{g}}=0^{\circ}$, the

TABLE I. Material properties for titanium, aluminum, and iron used for numerical results (Ref. 36).

\begin{tabular}{|c|c|c|c|c|c|c|c|c|}
\hline \multirow[b]{2}{*}{ Material } & \multirow{2}{*}{$\begin{array}{l}\text { Density } \\
\left(\mathrm{kg} / \mathrm{m}^{3}\right)\end{array}$} & \multicolumn{5}{|c|}{ Elastic Constants (GPa) } & \multicolumn{2}{|c|}{ Critical Angle (deg) } \\
\hline & & $c_{11}$ & $c_{12}$ & $c_{13}$ & $c_{33}$ & $c_{44}$ & $\theta_{\mathrm{I}}$ & $\theta_{\mathrm{II}}$ \\
\hline Titanium & 4506 & 160 & 90 & 66 & 181 & 46.5 & 15.97 & 32.01 \\
\hline Aluminum & 2700 & 108 & 62 & $c_{12}$ & $c_{11}$ & 28.3 & 14.92 & 32.21 \\
\hline Iron & 7874 & 230 & 135 & $c_{12}$ & $c_{11}$ & 117 & 15.99 & 29.55 \\
\hline
\end{tabular}

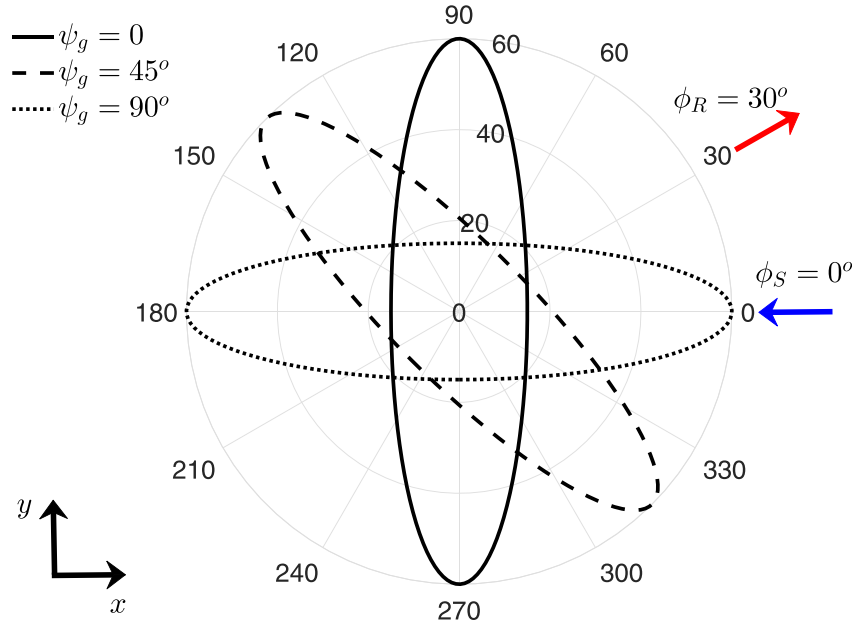

FIG. 3. (Color online) Polar angle notation in the $x y$-plane for grain elongation direction $\psi_{g}$, source transducer $\phi_{S}$ (depicted with an inward blue arrow), and receiving transducer $\phi_{R}$ (depicted with an outward red arrow).

time-dependent scattering response is calculated for a pulseecho (PE) configuration where $\phi_{S}=\phi_{R}=0^{\circ}$ and for a pitchcatch (PC) configuration where $\phi_{S}=0^{\circ}, \phi_{R}=90^{\circ}$. The results are depicted in Fig. 4. The responses are normalized by $V_{\max }^{2}$, the water path is set at $35 \mathrm{~mm}$, and $\Theta=45^{\circ}$ with the angles in the fluid $\left(\theta_{i}\right)$ adjusted according to Snell's law. First, consider the time dependence of the two configurations. A sharper response in time is observed in PC when compared with PE; the width in the time response is dictated by the overlap region of the beam profiles at the focus, which is largest in the PE configuration. The time dependence for different polar scattering angles, $\phi_{R}$, is similar to the PE configuration only for angles $\pm 5^{\circ}$ from $\phi_{S}=0^{\circ}$, then quickly converges to the time dependence shown for the PC configuration at all other angles, $\phi_{R}$. The narrower focal region also results in a later apparent arrival of the peak scattering amplitude. When comparing the different materials, the changes in arrival time result from the choice of a constant water path, which leads to small differences in the material path (or focal zone) due to wave speed differences between the solids. Second, consider the differences in scattering amplitudes. The scattering amplitude for the PE configuration is over 3 times larger than the PC configuration. Such a result is expected for $\psi_{g}=0^{\circ}$ because fewer grain boundaries are encountered when the wave scatters along the direction of elongation (i.e., when $\phi_{R}=90^{\circ}$ ). In agreement with longitudinal and mode-converted scattering results, the normalized time-dependent responses for different materials are similar with only a variation in amplitude. For the three materials considered, aluminum exhibits the highest amplitude followed by titanium and then iron. Although iron is more highly scattering due to its anisotropy, the amplitude of the scattering response is lowest due to losses at the interface, i.e., the factor $T_{T f} T_{f T} / R_{f f}$ is more than 20 times larger for aluminum than iron, and almost 4 times larger than titanium. In Secs. III B and III C, the peak scattering amplitude for aluminum is evaluated as a function of polar angle, elevation angle, and grain morphology. The results presented are expected to be similar for other materials. 

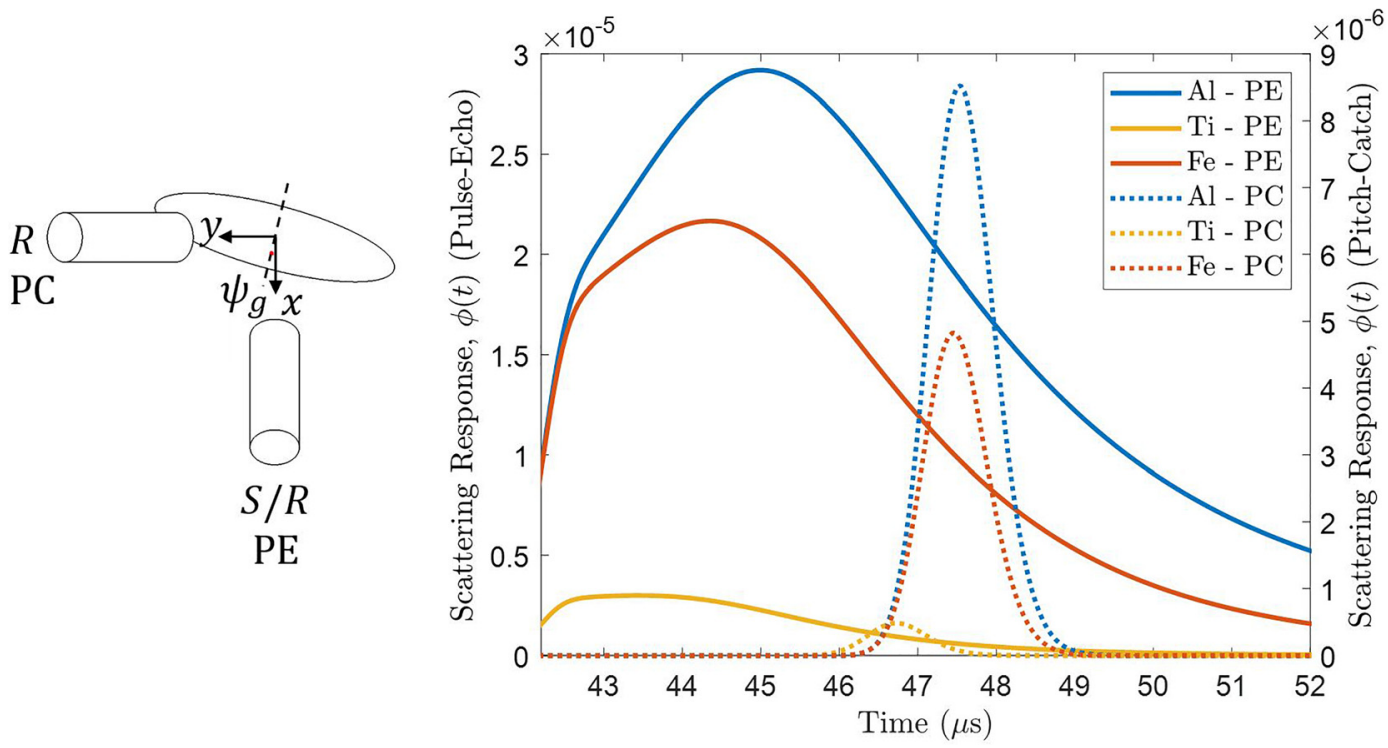

FIG. 4. (Color online) Time-dependent scattering response for three materials, aluminum, iron, and titanium with grain dimensions $a_{\mathrm{x}}=15 \mu \mathrm{m} ; a_{\mathrm{y}}=60 \mu \mathrm{m}$; $a_{\mathrm{z}}=30 \mu \mathrm{m}$, and $\psi_{\mathrm{g}}=0^{\circ}$. Two transducer configurations are depicted: pulse-echo (PE) where $\phi_{S}=\phi_{R}=0^{\circ}$ and pitch-catch (PC) where $\phi_{S}=0^{\circ}, \phi_{R}=90^{\circ}$.

\section{B. Polar angle dependence}

In this section, the maximum amplitude of the scattering response as a function of polar angle is studied. The source is fixed at $\phi_{S}=0^{\circ}$ and the receiver angle is varied from $\phi_{R}=0^{\circ}$ (PE) through various $\mathrm{PC}$ configurations back to $\phi_{R}=360^{\circ}$ (PE). Experimentally, measurement of the scattering response at arbitrary polar angles may require the use of special fixtures or configurations. These custom setups may result in slight errors in the positioning of the source and receiver, even when the intent is to maintain equal elevation angles. To address the effect of such a scenario, small variations in the elevation angle for the source $\theta_{i_{S}}$ and receiver $\theta_{i_{R}}$ are studied (depicted in Fig. 5). The angular dependence remains approximately equal for small variations in elevation angle. The differences are mainly observed in the amplitudes in select scattering directions for the given elongation direction $\left(\psi_{g}=0^{\circ}\right)$. The largest deviations from the $\theta_{i_{S}}=\theta_{i_{R}}$ case are observed around the backscatter $\left(\phi_{R}=0^{\circ}\right)$ and the forward scattering $\left(\phi_{R}=180^{\circ}\right)$ directions. In these cases, the variation can exceed $19 \%$. For other elongation directions, however, the error may manifest at different scattering angles. These results illustrate the importance of ensuring the incidence angles of the transducers are well controlled.

Assuming now that $\theta_{i_{S}}=\theta_{i_{R}}=\theta_{i}$, the effect of elevation angle on peak scattering amplitude for three elongation directions is given in Fig. 6. As expected, symmetry about

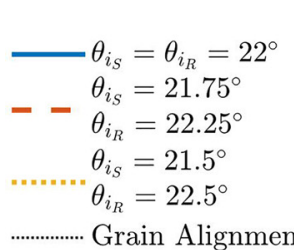

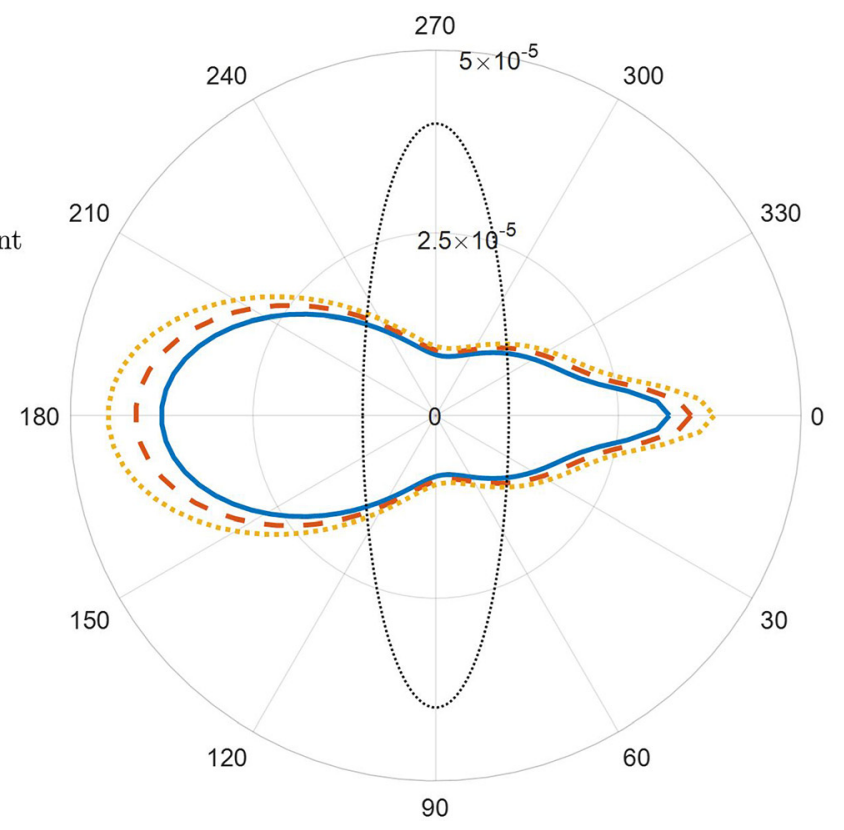

270

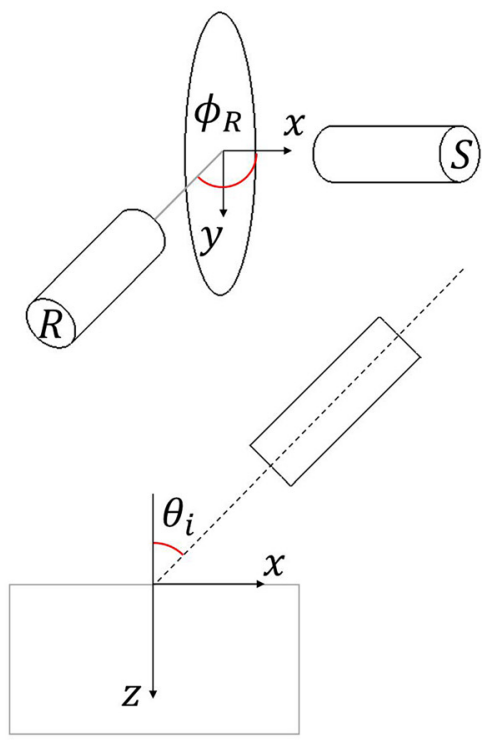

FIG. 5. (Color online) Effect of small variations in the elevation angle of the source $\left(\theta_{i_{S}}\right)$ and receiver $\left(\theta_{i_{R}}\right)$ on the peak amplitude of the shear scattering response in aluminum for a fixed grain elongation ratio $\left(a_{\mathrm{x}}=15 \mu \mathrm{m} ; a_{\mathrm{y}}=60 \mu \mathrm{m} ; a_{\mathrm{z}}=30 \mu \mathrm{m}\right)$ and direction $\left(\psi_{g}=0^{\circ}\right)$. The source is fixed at a polar angle $\phi_{S}=0^{\circ}$ and the amplitudes are given as a function of polar angle of the receiver $\phi_{R}$. 


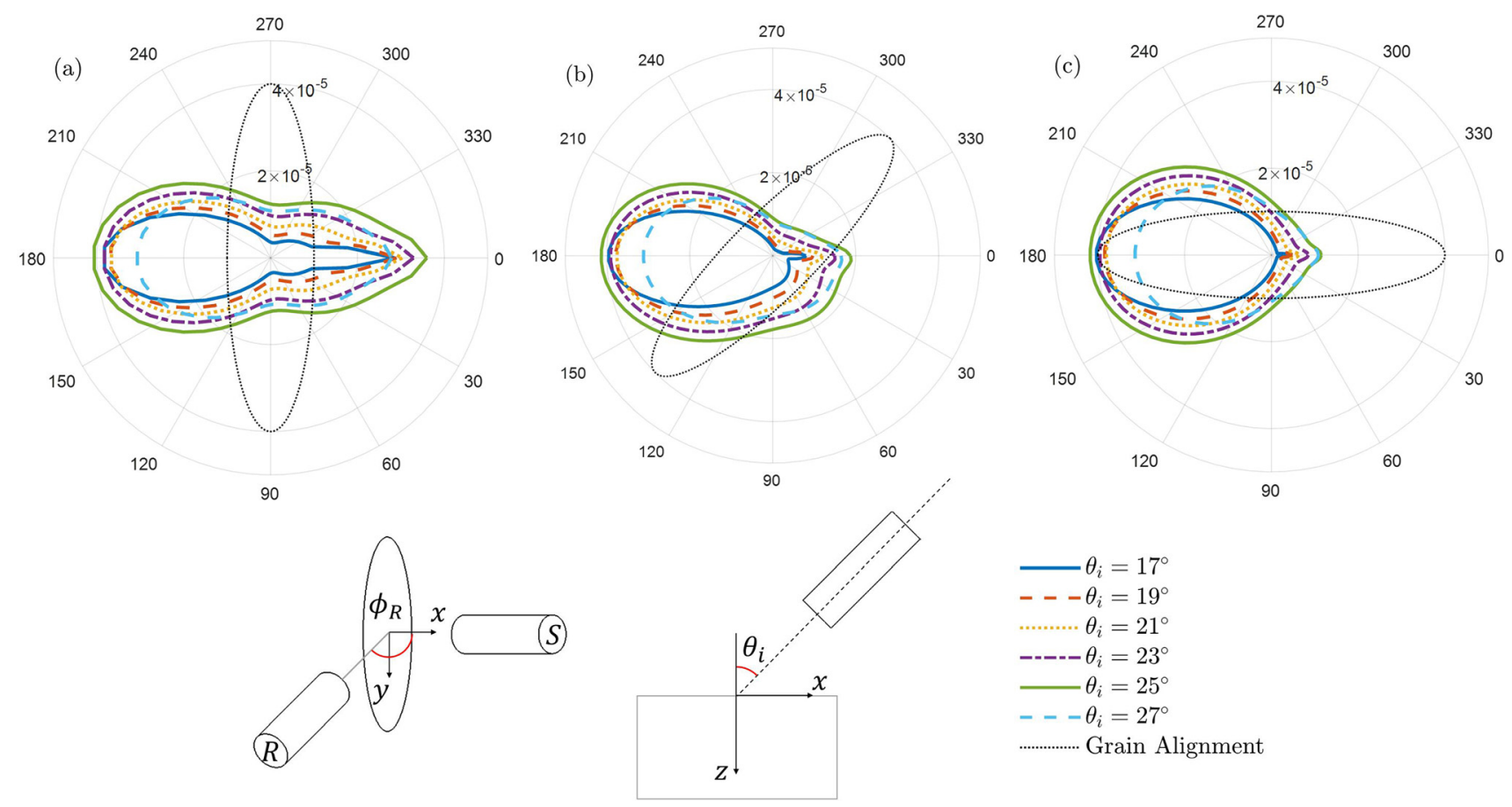

FIG. 6. (Color online) Peak amplitude of shear scattering response as a function of polar angle of the receiver $\phi_{R}$ (with $\phi_{S}=0$ ) for a fixed grain elongation ratio $\left(a_{\mathrm{x}}=15 \mu \mathrm{m} ; a_{\mathrm{y}}=60 \mu \mathrm{m} ; a_{\mathrm{z}}=30 \mu \mathrm{m}\right)$ and varying elevation angle $\theta_{i}$ for aluminum.

$\phi_{R}=0-180^{\circ}$ is observed when the grain is elongated along the $x$ or $y$ directions $\left(\psi_{g}=0^{\circ}\right.$ and $\left.\psi_{g}=90^{\circ}\right)$. When considering the response as a function of polar angle, $\phi_{R}$, a local maximum is observed for the PE transducer configuration (corresponding with backscattered energy) for all grain orientations. This local maximum becomes more prominent at the larger elevation angles, $\theta_{i}$. Furthermore, the PE configuration displays the largest change in amplitude for different elongation directions, regardless of elevation angle. Conversely, the forward scattering (FS) energy (when $\phi_{R}=180^{\circ}$ ) has the largest magnitude regardless of elongation direction but is the least sensitive to the elongation direction. A local minimum is observed for $\phi_{R}=90^{\circ}$ when $\psi_{g}=0^{\circ}$, which corresponds with scattering along the direction of primary elongation. When the grain is rotated with respect to the transducers, however, the local minimum shifts and becomes dependent on elevation angle $\theta_{i}$. Considering the scattering amplitude as a function of elevation angle $\theta_{i}$, multiple local maxima are observed, most evidently for the PE and FS transducer configurations. To understand this phenomenon further, the response as a function of elevation angle for select transducer configurations is studied.

Figures 7-9 show results for (a) a PE configuration, (b) a PC configuration with $\phi_{R}=90^{\circ}$, and (c) a PC forward
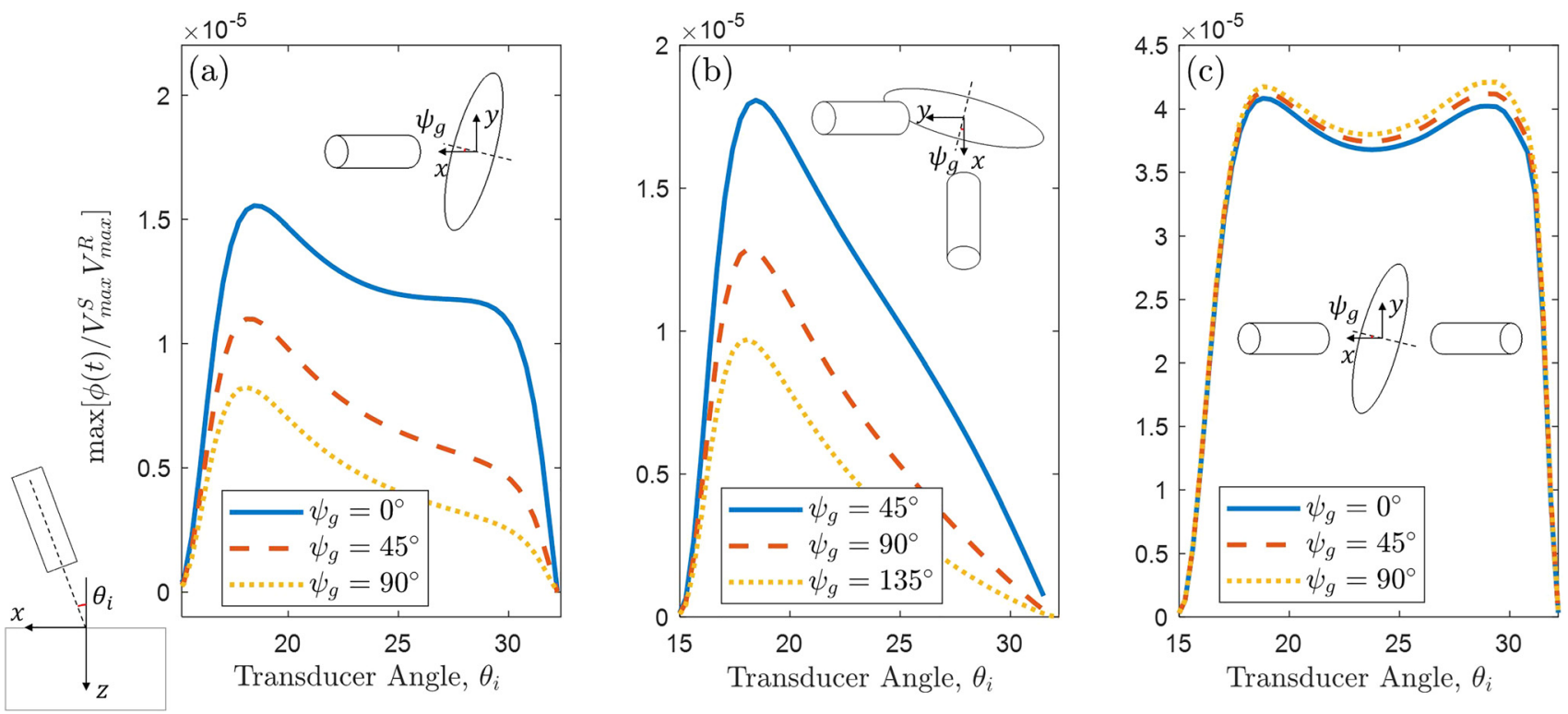

FIG. 7. (Color online) Peak amplitude of the shear scattering response with $\phi_{S}=0^{\circ}$ and (a) $\phi_{R}=0^{\circ}$, (b) $\phi_{R}=90^{\circ}$, and (c) $\phi_{R}=180^{\circ}$ as a function of elevation angle $\theta_{i}$ for a fixed grain elongation ratio $\left(a_{\mathrm{x}}=15 \mu \mathrm{m} ; a_{\mathrm{y}}=60 \mu \mathrm{m} ; a_{\mathrm{z}}=30 \mu \mathrm{m}\right)$ and various grain elongation directions $\psi_{\mathrm{g}}$ in aluminum. 

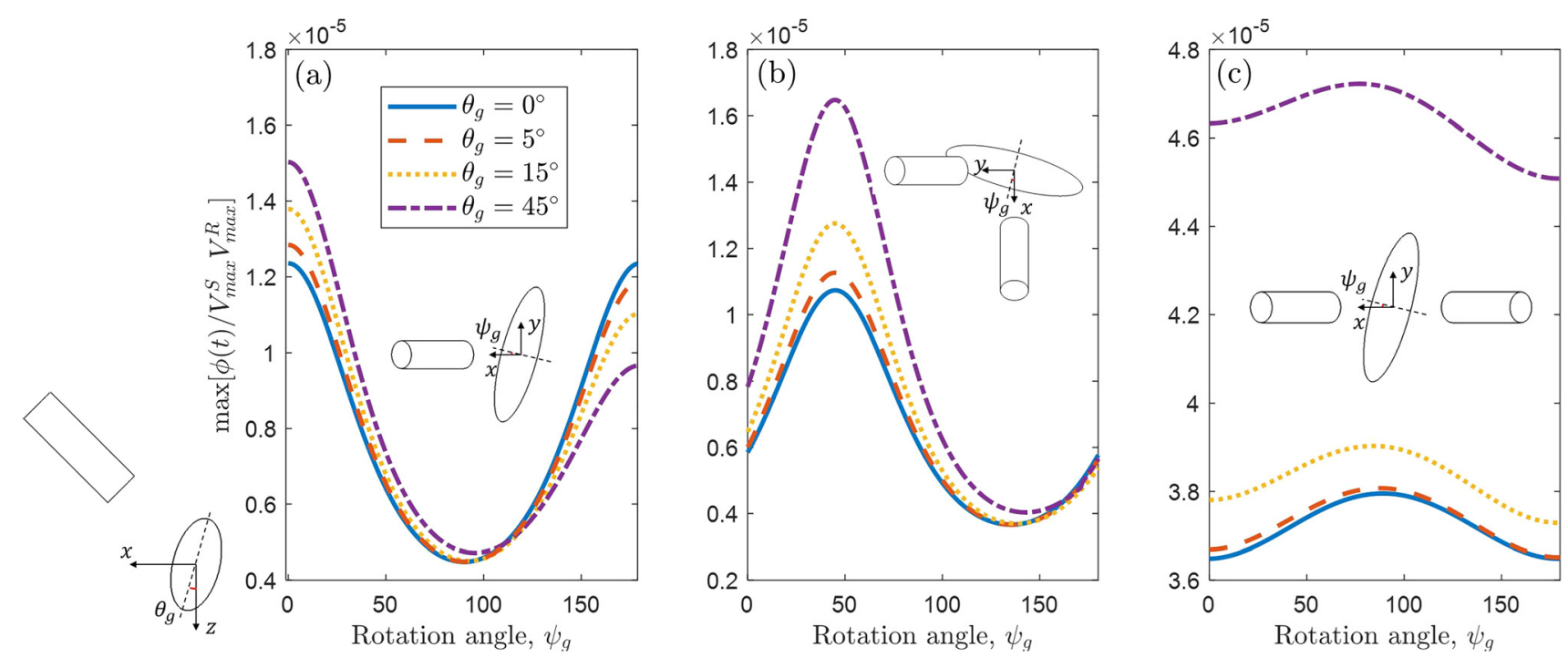

FIG. 8. (Color online) Peak amplitude of the shear scattering response with $\phi_{S}=0^{\circ}$ and (a) $\phi_{R}=0^{\circ}$, (b) $\phi_{R}=90^{\circ}$, and (c) $\phi_{R}=180^{\circ}$ as a function of rotation angle $\theta_{g}$ for a fixed grain elongation ratio $\left(a_{\mathrm{x}}=15 \mu \mathrm{m} ; a_{\mathrm{y}}=60 \mu \mathrm{m} ; a_{\mathrm{z}}=30 \mu \mathrm{m}\right)$ and various grain elongation directions $\psi_{\mathrm{g}}$ in aluminum.

scattering (FS) configuration where $\phi_{R}=180^{\circ}$. The maximum scattering amplitude as a function of elevation angle is given in Fig. 7. For all scattering scenarios, a sharp increase in the amplitude of the response occurs after the first critical angle, $\theta_{I}$. For PE in Fig. 7(a), the amplitude slightly decreases before reaching a second local maximum near the second critical angle, $\theta_{I I}$. The amplitude then sharply drops to zero at $\theta_{I I}$. This response follows a trend similar to the square of the transmission-reflection coefficient ratio given by $T_{f T} T_{T f} / R_{f f}$ which multiplies the scattering response. The FS response in Fig. 7(c) displays a similar trend with a smaller change in the amplitude of the response as a function of incident angle. Figure 7(c) also illustrates how small the change in amplitude is for different grain orientations. Contrary to PE, the FS amplitude is largest when the wave propagates and scatters along the grain elongation direction. For PC in Fig. 7(b), the response most resembles the mode- converted scattering response dependence on angle of incidence. ${ }^{28}$ The decrease in amplitude is gradual after the maximum near, $\theta_{I}$. Note that the grain orientation angles depicted are $\psi_{g}=45^{\circ}, 90^{\circ}$, and $135^{\circ}$ because the symmetry in the response for this transducer configuration is about $\psi_{g}=45^{\circ}$ rather than $\psi_{g}=0^{\circ}$ or $90^{\circ}$ as shown later in Fig. 8. The behavior near $\theta_{I}$ and $\theta_{I I}$ may be of interest although it is expected to be more complex due to contributions from other modes of scattering; hence, it is beyond the scope of this article.

\section{Grain morphology dependence}

Next, the effect of grain rotation about axis $Y_{g}$ is studied, hereon referred to as grain tilt. The maximum amplitude of the response as a function of angle $\psi_{g}$ is given in Fig. 8 for various tilt angles, $\theta_{g}$. The response for PE in Fig. $8(\mathrm{a})$
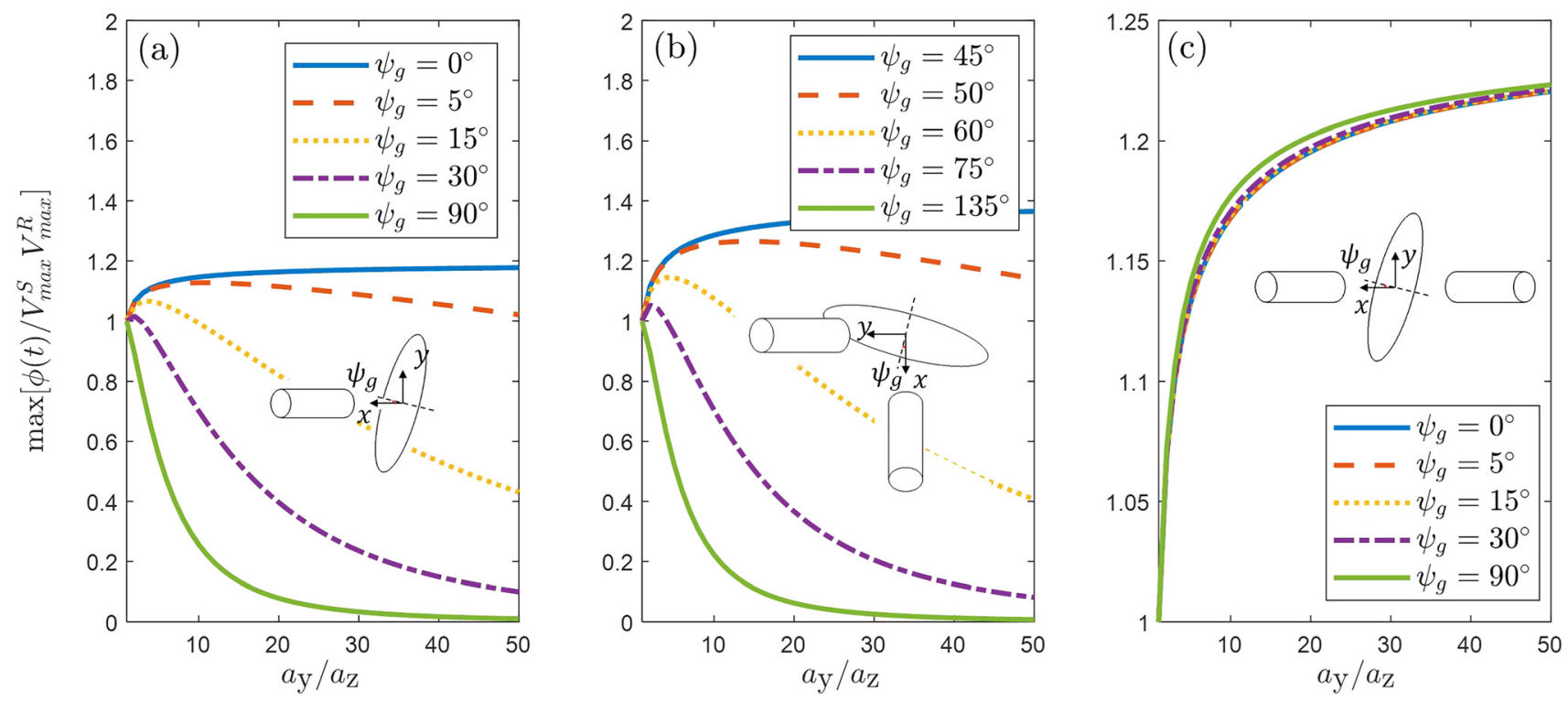

FIG. 9. (Color online) Normalized peak amplitude of the shear scattering response with $\phi_{S}=0^{\circ}$ and (a) $\phi_{R}=0^{\circ}$, (b) $\phi_{R}=90^{\circ}$, and (c) $\phi_{R}=180^{\circ}$ as a function of aspect ratio of the elongated grains (assuming $a_{\mathrm{x}}=a_{\mathrm{z}}$ and a constant grain volume of $V=4500 \pi \mu \mathrm{m}^{3}$ ) in aluminum. 
follows the same trend as the mode-converted scattering response; ${ }^{28}$ for no grain rotation, there is symmetry about $\psi_{g}=90^{\circ}$ that is disrupted in the presence of grain tilt. Such an effect is anticipated by the fact that the effective crosssectional length along the direction of propagation is modified when the grains are tilted. Additionally, the absolute amplitude of scattering is larger for the PE shear scattering case than it is for mode-converted scattering. ${ }^{28}$ Some of the contributing factors include changes in the transmission coefficients at the fluid/solid interface and differences in the propagation distances when maintaining a constant focal depth. For PC in Fig. 8(b), the response is symmetric about $\psi_{g}=45^{\circ}$ which corresponds with the maximum amplitude given the smallest cross-sectional area along the wave propagation direction. The presence of grain tilt increases this maximum amplitude more significantly than for the PE configuration. For FS in Fig. 8(c), as previously mentioned, the maximum is less prominent and occurs at $\psi_{g}=90^{\circ}$ which corresponds with elongation along the direction of wave propagation. For FS, when the grains are rotated about $Y_{g}$, not only is the symmetry of the response about $\psi_{g}=90^{\circ}$ disrupted, but the magnitude of the scattering also increases significantly. However, note that for a fixed grain rotation $\theta_{g}$, the variation in scattering amplitude as a function of $\psi_{g}$ is still significantly smaller than for the other two transducer configurations. Note that for the largest grain tilt, $\theta_{g}=45^{\circ}$, in the forward scattering configuration selected $\left(\Theta=45^{\circ}\right)$, the incident wave propagates along $a_{\mathrm{x}}$ and scatters along $a_{\mathrm{z}}$. Although it is apparent that for these fixed grain dimensions the ratio from maximum to minimum scattering amplitude is largest for the $\phi_{R}=90^{\circ}$ configuration, the effect of grain elongation on scattering amplitude is more clearly depicted in Fig. 9.

In order to study the effect of elongation ratio, grains with circular cross-sections (i.e., $a_{\mathrm{x}}=a_{\mathrm{z}}$ ) are elongated in the $\hat{\mathbf{Y}}_{\mathrm{g}}$ direction maintaining a constant grain volume $\left(V=4500 \pi \mu \mathrm{m}^{3}\right)$. The maximum amplitude of the response is normalized by the maximum amplitude of the response for equiaxed grains $\left(a_{\mathrm{x}}=a_{\mathrm{y}}=a_{\mathrm{z}}\right)$. Various rotation angles $\psi_{\mathrm{g}}$ between $0^{\circ}$ and $90^{\circ}$ are considered for PE and FS (the circular cross-section implies symmetry about $\psi_{g}=90^{\circ}$ ); and values between $45^{\circ}$ and $135^{\circ}$ are considered for the PC setup. As shown in the previous two examples, the FS response in Fig. 9(c) has negligible dependence on grain rotation. For FS, the deviation from the equiaxed grain response to $a_{\mathrm{y}}=50 a_{\mathrm{z}}$ is consistently around $20 \%$ for all values of $\psi_{g}$ considered. The PE and PC relative amplitudes have a trend similar to the mode-converted scattering case; increased elongation increases the amplitude ratio for perpendicular scattering directions $\left(\psi_{g}=0{ }^{\circ} / \psi_{g}=90^{\circ}\right.$ and $\psi_{g}=45^{\circ} / \psi_{g}=135^{\circ}$ ). The scattering amplitude when the receiving transducer is perpendicular to the direction of elongation $\left(\psi_{g}=90^{\circ}\right.$ or $\left.\psi_{g}=135^{\circ}\right)$ rapidly decreases as a function of aspect ratio. In addition, the difference in scattering amplitude for $\psi_{g}$ angles approaching scattering perpendicular to elongation quickly decreases as a function of aspect ratio. Therefore, greater measurement sensitivity to elongation exists for smaller ranges of $\psi_{g}$ when elongation ratios are large. Lastly, the PC configuration in Fig. 9(b) exhibits the largest deviation from the equiaxed grain scattering amplitude nearing $40 \%$ for $a_{\mathrm{y}}=50 a_{\mathrm{z}}$.

\section{SUMMARY}

In this article, a generalized scattering model was derived for arbitrary configurations of the source and receiving transducers. The scattering coefficient was explicitly given for statistically isotropic solids with aligned ellipsoidal grains. A supplementary MATLAB code is provided to calculate the elastic covariance for crystallites of any symmetry class, along with the inner products for random wave propagation and displacement directions. ${ }^{37}$ Numerical results were presented for out-ofplane shear-to-shear scattering for $\mathrm{Al}, \mathrm{Fe}$, and Ti. Different materials were shown to have comparable scattering responses with only amplitude variations, which were dominated by the reflection and transmission coefficients at the interface. The importance of precise transducer placement was illustrated by evaluating the amplitude of the scattering response; for $\pm 0.5^{\circ}$ variations in elevation, amplitude differences approaching $20 \%$ were observed. Next, the effects of elevation angle and grain morphology were evaluated. Depending on the transducer configuration, varying sensitivity to grain orientation and size were observed. One advantage of this generalized model is the ability to define experimental configurations with increased sensitivity to microstructural parameters of interest. The given formulation, which separates the scattering coefficient from the parameters pertaining to transducer placement, provides a starting point for studying increasingly complex microstructures. To this end, the modification of the scattering coefficient to include macroscopic texture would be a natural continuation of the present work.

\section{APPENDIX A: SINGLE GAUSSIAN BEAM PARAMETERS}

Restricting the analysis to planar samples, the single Gaussian beam parameters are given by ${ }^{25,30}$

$$
\begin{aligned}
\frac{1}{q_{\xi}\left(Z_{\xi}\right)} & =\frac{1}{R_{\xi}\left(Z_{\xi}\right)}-i \frac{2}{k_{f} w_{\xi}^{2}\left(Z_{\xi}\right)}, \\
q_{1 \xi}\left(Z_{\xi}\right) & =\frac{\cos ^{2} \theta_{r_{\xi}}}{\cos ^{2} \theta_{i_{\xi}}}\left[q_{\xi}(0)+z_{f \xi}\right]+\frac{c_{\chi}}{c_{f}} Z_{\xi}, \\
q_{2 \xi}\left(Z_{\xi}\right) & =q_{\xi}(0)+z_{f \xi}+\frac{c_{\chi}}{c_{f}} Z_{\xi} .
\end{aligned}
$$

$Z_{S}$ and $Z_{R}$ are the source and receiver propagation axes, respectively. $\theta_{i}$ is the angle of incidence of each transducer in the fluid and $\theta_{r}$ is the refraction angle calculated using Snell's law $\left(\theta_{r_{\xi}}=\Theta_{\xi}\right.$ in Fig. 2). $R_{\xi}(0)=-F_{\xi}$ and $w_{\xi}(0)=w_{0 \xi}=0.7517 a_{\xi}$ are the initial radius and beam width of the wavefront, respectively, where $F$ is the focal length of the transducer in the fluid and $a$ is the nominal radius of the transducer element. ${ }^{30} k_{f}=\omega_{0} / c_{f}$ is the wave number of the immersion fluid at the center frequency of the transducer. The widths of the single Gaussian beam can now be written as

$$
\begin{aligned}
& w_{1}^{2}\left(Z_{\xi}\right)=-\frac{2}{k_{f} \operatorname{Im}\left(1 / q_{1 \xi}\left(Z_{\xi}\right)\right)}, \\
& w_{2}^{2}\left(Z_{\xi}\right)=-\frac{2}{k_{f} \operatorname{Im}\left(1 / q_{2 \xi}\left(Z_{\xi}\right)\right)} .
\end{aligned}
$$


Note that these expressions assume a circular cross-section for the beam profile, which becomes ellipsoidal for obliqueincidence. $^{27}$

\section{APPENDIX B: SPATIAL CORRELATION FUNCTION}

The spatial correlation function $\eta$ represents the probability that two randomly chosen positions lie within a given grain. The spatial Fourier transform of the correlation function can be written in terms of the unit vectors $\hat{\mathbf{k}}_{0}$ and $\hat{\mathbf{k}}_{0}^{\prime}$, given in Fig. 1, or in terms of the global coordinate system as

$$
\tilde{\eta}(\mathbf{q})=\tilde{\eta}\left(k^{\prime} \hat{\mathbf{k}}_{0}^{\prime}-k \hat{\mathbf{k}}_{0}\right)=\tilde{\eta}\left(q_{x} \hat{\mathbf{x}}+q_{y} \hat{\mathbf{y}}+q_{z} \hat{\mathbf{z}}\right) .
$$

In this article, the case of ellipsoidal grains is considered, for which the correlation function reduces to ${ }^{11,16,28,34}$

$$
\tilde{\eta}=\tilde{\eta}(\mathbf{q})=\frac{a_{\mathrm{x}} a_{\mathrm{y}} a_{\mathrm{z}}}{\pi^{2}\left(1+a_{\mathrm{x}}^{2} q_{x}^{2}+a_{\mathrm{y}}^{2} q_{y}^{2}+a_{\mathrm{z}}^{2} q_{z}^{2}\right)^{2}},
$$

where $a_{\mathrm{x}}, a_{\mathrm{y}}$, and $a_{\mathrm{z}}$ define the radii of the ellipsoid in the $x$, $y$, and $z$ directions, respectively. This equation simplifies to the case of equiaxed grains when $a_{\mathrm{x}}=a_{\mathrm{y}}=a_{\mathrm{z}}=L$. The directions of unit vectors $\hat{\mathbf{k}}_{0}$ and $\hat{\mathbf{k}}_{0}^{\prime}$ correspond with $Z_{S}$ and $-Z_{R}$, respectively, as seen in Fig. 2 , such that

$$
\begin{aligned}
q_{x} & =\left(k^{\prime} \hat{\mathbf{k}}_{0}^{\prime}-k \hat{\mathbf{k}}_{0}\right) \cdot \hat{\mathbf{x}} \\
& =k^{\prime} \cos \phi_{R} \sin \Theta_{R}+k \cos \phi_{S} \sin \Theta_{S}, \\
q_{y} & =\left(k^{\prime} \hat{\mathbf{k}}_{0}^{\prime}-k \hat{\mathbf{k}}_{0}\right) \cdot \hat{\mathbf{y}} \\
& =k^{\prime} \sin \phi_{R} \sin \Theta_{R}+k \sin \phi_{S} \sin \Theta_{S}, \text { and } \\
q_{z} & =\left(k^{\prime} \hat{\mathbf{k}}_{0}^{\prime}-k \hat{\mathbf{k}}_{0}\right) \cdot \hat{\mathbf{z}}=-k^{\prime} \cos \Theta_{R}-k \cos \Theta_{S},
\end{aligned}
$$

which simplifies to the expressions given by Arguelles et $a .^{28}$ when $\phi_{S}=\phi_{R}=0^{\circ}$. Here $k=\omega_{0} / c_{\chi}$ is the wave number of the incident wave and $k^{\prime}=\omega_{0} / c_{\chi^{\prime}}$ is the wave

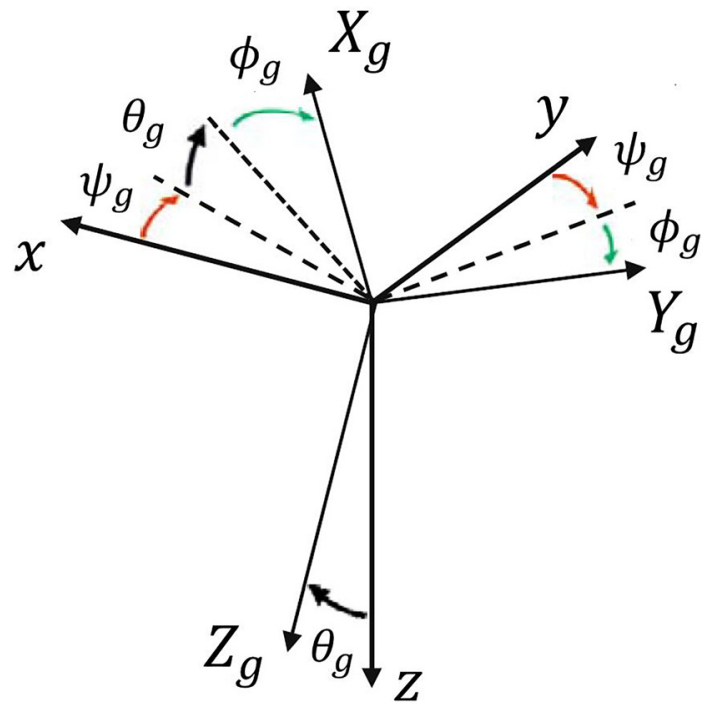

FIG. 10. (Color online) Euler angles used to define the grain coordinate system relative to the fixed reference frame.

number of the scattered wave, where $\chi$ represents the wave mode (longitudinal or shear). $\Theta_{\xi}$ and $\phi_{\xi}$ are the angles of the source and receiver with respect to the global coordinate system given by Eq. (5). A new coordinate system is defined for the ellipsoidal grains in order to allow arbitrary directions of elongation with respect to the transducer configuration; this step is necessary for PC transducer configurations where rotation of the transducers is not sufficient to describe tilt or out-of-plane grain rotation. The relation between the global axes $(x, y, z)$ and the grain axes $\left(X_{g}, Y_{g}, Z_{g}\right)$ can be specified using three Euler angles $\psi_{g}, \theta_{g}$, and $\phi_{g}$, as shown in Fig. 10. For the rotation convention used, $\psi_{g}$ defines rotation about $\hat{\mathbf{z}}$, $\theta_{g}$ defines rotation about $\hat{\mathbf{Y}}_{\mathbf{g}}$, and $\phi_{g}$ defines rotation about $\hat{\mathbf{Z}}_{\mathrm{g}}$. Note that there is redundancy in one angle when both the grain and transducers are allowed to rotate in-plane, but this definition facilitates illustration of numerical results. Now, the coordinate transformation from $\mathbf{X}_{\mathbf{g}}$ to $\mathbf{x}$ can be written as $\mathbf{X}_{\mathrm{g}}=\mathbf{r X}$ where the transformation matrix $\mathbf{r}$ is given by

$$
\mathbf{r}=\left(\begin{array}{ccc}
\cos \psi_{g} \cos \theta_{g} \cos \phi_{g}-\sin \psi_{g} \sin \phi_{g} & \sin \psi_{g} \cos \theta_{g} \cos \phi_{g}+\cos \psi_{g} \sin \phi_{g} & -\sin \theta_{g} \cos \phi_{g} \\
-\cos \psi_{g} \cos \theta_{g} \sin \phi_{g}-\sin \psi_{g} \cos \phi_{g} & -\sin \psi_{g} \cos \theta_{g} \sin \phi_{g}+\cos \psi_{g} \cos \phi_{g} & \sin \theta_{g} \sin \phi_{g} \\
\cos \psi_{g} \sin \theta_{g} & \sin \psi_{g} \sin \theta_{g} & \cos \theta_{g}
\end{array}\right)
$$

The vector q in Eq. (B1) can then be modified and written with respect to the coordinate system of the elongated grains, so that $\mathbf{q}=q_{X_{g}} \hat{\mathbf{X}}_{\mathbf{g}}+q_{Y_{g}} \hat{\mathbf{Y}}_{\mathbf{g}}+q_{Z_{\mathbf{g}}} \hat{\mathbf{Z}}_{\mathbf{g}}$. Because $\mathbf{X}_{\mathbf{g}}=\mathbf{r X}$ $\left(X_{g_{i}}=r_{i j} x_{j}\right)$, the following expressions are obtained:

$$
\begin{aligned}
& q_{X_{g}}=\mathbf{q} \cdot \hat{\mathbf{X}}_{\mathbf{g}}=r_{11} q_{x}+r_{12} q_{y}+r_{13} q_{z}, \\
& q_{Y_{g}}=\mathbf{q} \cdot \hat{\mathbf{Y}}_{\mathbf{g}}=r_{21} q_{x}+r_{22} q_{y}+r_{23} q_{z}, \\
& q_{Z_{g}}=\mathbf{q} \cdot \hat{\mathbf{Z}}_{\mathbf{g}}=r_{31} q_{x}+r_{32} q_{y}+r_{33} q_{z},
\end{aligned}
$$

where the correlation function is now given by

$$
\tilde{\eta}(\mathbf{q})=\frac{a_{\mathrm{x}} a_{\mathrm{y}} a_{\mathrm{z}}}{\pi^{2}\left(1+a_{\mathrm{x}}^{2} q_{X_{g}}^{2}+a_{\mathrm{y}}^{2} q_{Y_{g}}^{2}+a_{\mathrm{z}}^{2} q_{Z_{g}}^{2}\right)^{2}} .
$$

This form of the correlation function was first given by Arguelles et al. ${ }^{28}$ for an in-plane PC transducer configuration. Note that the presence of grain size distributions is ignored, which may have a measurable effect on the scattering response. ${ }^{35}$ 


\section{APPENDIX C: COVARIANCE INNER PRODUCT}

Because the medium is assumed to be statistically isotropic, the inner products in Eq. (12) are defined assuming random crystallographic orientations. In order to define the inner products, the displacement vectors for the different mode types must be defined. The graphical representation for these vectors is given in Fig. 2. For the incident wave, the propagation direction $\hat{\mathbf{k}}$ corresponds with $Z_{S}$ which coincides with the displacement direction for a longitudinal wave $\hat{\mathbf{e}}_{L}$. The displacement of the shear vertical wave $\hat{\mathbf{e}}_{S V}$ is set to correspond with $X_{S}$ and the displacement of the shear horizontal wave $\hat{\mathbf{e}}_{S H}$ is set to correspond with $-Y_{S}$, yielding

$$
\begin{aligned}
& \hat{\mathbf{k}}=\hat{\mathbf{e}}_{L}=-\cos \phi_{S} \sin \Theta_{S} \hat{\mathbf{x}}-\sin \phi_{S} \sin \Theta_{S} \hat{\mathbf{y}}+\cos \Theta_{S} \hat{\mathbf{z}}, \\
& \hat{\mathbf{e}}_{S V}=\cos \phi_{S} \cos \Theta_{S} \hat{\mathbf{x}}+\sin \phi_{S} \cos \Theta_{S} \hat{\mathbf{y}}+\sin \Theta_{S} \hat{\mathbf{z}}, \\
& \hat{\mathbf{e}}_{S H}=\sin \phi_{S} \hat{\mathbf{x}}-\cos \phi_{S} \hat{\mathbf{y}}
\end{aligned}
$$

For the scattered wave, the propagation direction $\hat{\mathbf{k}}^{\prime}$ and displacement direction of a longitudinal wave $\hat{\mathbf{e}}_{L}^{\prime}$ correspond with $-Z_{R}$. The displacement of the shear vertical wave $\hat{\mathbf{e}}_{S V}^{\prime}$ corresponds with $X_{R}$, and the displacement of the shear horizontal wave $\hat{\mathbf{e}}_{S H}^{\prime}$ corresponds with $-Y_{R}$, yielding

$$
\begin{aligned}
& \hat{\mathbf{k}}^{\prime}=\hat{\mathbf{e}}_{L}^{\prime}=\cos \phi_{R} \sin \Theta_{R} \hat{\mathbf{x}}+\sin \phi_{R} \sin \Theta_{R} \hat{\mathbf{y}}-\cos \Theta_{R} \hat{\mathbf{z}}, \\
& \hat{\mathbf{e}}_{S V}^{\prime}=\cos \phi_{R} \cos \Theta_{R} \hat{\mathbf{x}}+\sin \phi_{R} \cos \Theta_{R} \hat{\mathbf{y}}+\sin \Theta_{R} \hat{\mathbf{z}}, \\
& \hat{\mathbf{e}}_{S H}^{\prime}=\sin \phi_{R} \hat{\mathbf{x}}-\cos \phi_{R} \hat{\mathbf{y}} .
\end{aligned}
$$

The inner product expressions for arbitrary wave propagation directions and arbitrary single crystal symmetry can be calculated using the supplementary MATLAB code. ${ }^{37}$

${ }^{1}$ J. E. Gubernatis, E. Domany, and J. A. Krumhansl, "Formal aspects of the theory of the scattering of ultrasound by flaws in elastic materials," J. Appl. Phys. 48(7), 2804-2811 (1977).

${ }^{2}$ J. E. Gubernatis, E. Domany, J. A. Krumhansl, and M. Huberman, "The born approximation in the theory of the scattering of elastic waves by flaws," J. Appl. Phys. 48(7), 2812-2819 (1977).

${ }^{3} \mathrm{G}$. S. Kino, "The application of reciprocity theory to scattering of acoustic waves by flaws," J. Appl. Phys. 49(6), 3190-3199 (1978).

${ }^{4}$ B. A. Auld, "General electromechanical reciprocity relations applied to the calculation of elastic wave scattering coefficients," Wave Motion 1(1), 3-10 (1979).

${ }^{5}$ R. B. Thompson and T. A. Gray, "A model relating ultrasonic scattering measurements through liquid-solid interfaces to unbounded medium scattering amplitudes," J. Acoust. Soc. Am. 74(4), 1279-1290 (1983).

${ }^{6}$ J. H. Rose, "Ultrasonic backscattering from polycrystalline aggregates using time-domain linear response theory," in Review of Progress in Quantitative Nondestructive Evaluation (Springer, New York, 1991), Vol. 10B, pp. 1715-1720.

${ }^{7}$ F. J. Margetan, T. A. Gray, and R. B. Thompson, "A technique for quantitatively measuring microstructurally induced ultrasonic noise," in Review of Progress in Quantitative Nondestructive Evaluation (Springer, New York, 1991), Vol. 10B, pp. 1721-1728.

${ }^{8}$ F. J. Margetan, R. B. Thompson, and I. Yalda-Mooshabad, "Backscattered microstructural noise in ultrasonic toneburst inspections," J. Nondestruct. Eval. 13(3), 111-136 (1994).

${ }^{9}$ F. J. Margetan, R. B. Thompson, and I. Yalda-Mooshabad, "Modeling ultrasonic microstructural noise in titanium alloys," in Review of Progress in Quantitative Nondestructive Evaluation (Springer, New York, 1993), Vol. 12, pp. 1735-1742.

${ }^{10}$ F. J. Margetan, K. Y. Han, I. Yalda-Mooshabad, S. Goettsch, and R. B. Thompson, "The practical application of grain noise models in titanium billets and forgings," in Review of Progress in Quantitative Nondestructive Evaluation (Springer, New York, 1995), Vol. 14, pp. 2129-2136.

${ }^{11}$ Y. K. Han and R. B. Thompson, "Ultrasonic backscattering in duplex microstructures: Theory and application to titanium alloys," Metal. Mater. Trans. A 28(1), 91-104 (1997).

${ }^{12}$ Y. K. Han, R. B. Thompson, I. Yalda, F. J. Margetan, A. J. Anderson, M. Hirao, and J. Root, "Effect of texture on ultrasonic backscattering coefficient in pure titanium plate," in Review of Progress in Quantitative Nondestructive Evaluation (Springer, New York, 1996), Vol. 15, pp. $1685-1691$.

${ }^{13}$ R. J. Roe, "Description of crystallite orientation in polycrystalline materials. III. General solution to pole figure inversion,” J. Appl. Phys. 36(6), 2024-2031 (1965).

${ }^{14} \mathrm{Y}$. Li and R. B. Thompson, "Relations between elastic constants cij and texture parameters for hexagonal materials," J. Appl. Phys. 67(5), 2663-2665 (1990).

${ }^{15}$ F. J. Margetan, L. Yu, and R. B. Thompson, "Computation of grainnoise scattering coefficients for ultrasonic pitch/catch inspections of metals," in Review of Progress in Quantitative Nondestructive Evaluation (AIP Publishing, Melville, NY, 2005), Vol. 760, pp. 1300-1307.

${ }^{16}$ O. I. Lobkis and S. I. Rokhlin, "Characterization of polycrystals with elongated duplex microstructure by inversion of ultrasonic backscattering data," Appl. Phys. Lett. 96(16), 161905 (2010).

${ }^{17}$ O. I. Lobkis, L. Yang, J. Li, and S. I. Rokhlin, "Ultrasonic backscattering in polycrystals with elongated single phase and duplex microstructures," Ultrasonics 52(6), 694-705 (2012).

${ }^{18} \mathrm{G}$. Sha, "Correlation of elastic wave attenuation and scattering with volumetric grain size distribution for polycrystals of statistically equiaxed grains," Wave Motion 83, 102-110 (2018).

${ }^{19}$ L. Yang, J. Li, O. I. Lobkis, and S. I. Rokhlin, "Ultrasonic propagation and scattering in duplex microstructures with application to titanium alloys," J. Nondestruct. Eval. 31(3), 270-283 (2012).

${ }^{20} \mathrm{~L}$. Yang, J. Li, and S. I. Rokhlin, "Ultrasonic scattering in polycrystals with orientation clusters of orthorhombic crystallites," Wave Motion 50(8), 1283-1302 (2013).

${ }^{21} \mathrm{~J}$. Li, L. Yang, and S. I. Rokhlin, "Effect of texture and grain shape on ultrasonic backscattering in polycrystals," Ultrasonics 54(7), 1789-1803 (2014).

${ }^{22} \mathrm{~J}$. Li and S. I. Rokhlin, "Elastic wave scattering in random anisotropic solids," Int. J. Solids Struct. 78-79, 110-124 (2016).

${ }^{23} \mathrm{G}$. Sha, "Explicit backscattering coefficient for ultrasonic wave propagating in hexagonal polycrystals with fiber texture," J. Nondestruct. Eval. 37(3), 37-51 (2018).

${ }^{24}$ G. Ghoshal, J. A. Turner, and R. L. Weaver, "Wigner distribution of a transducer beam pattern within a multiple scattering formalism for heterogeneous solids," J. Acoust. Soc. Am. 122(4), 2009-2021 (2007).

${ }^{25} \mathrm{G}$. Ghoshal and J. A. Turner, "Diffuse ultrasonic backscatter at normal incidence through a curved interface," J. Acoust. Soc. Am. 128(6), 3449-3458 (2010).

${ }^{26} \mathrm{P}$. Hu, C. M. Kube, L. W. Koester, and J. A. Turner, "Mode-converted diffuse ultrasonic backscatter," J. Acoust. Soc. Am. 134(2), 982-990 (2013).

${ }^{27} \mathrm{P}$. Hu and J. A. Turner, "Transverse-to-transverse diffuse ultrasonic scattering," J. Acoust. Soc. Am. 142(2), 1112-1120 (2017).

${ }^{28}$ A. P. Arguelles, C. M. Kube, P. Hu, and J. A. Turner, "Mode-converted ultrasonic scattering in polycrystals with elongated grains," J. Acoust. Soc. Am. 140(3), 1570-1580 (2016).

${ }^{29}$ L. Schmerr and J-S. Song, Ultrasonic Nondestructive Evaluation Systems (Springer, New York, 2007).

${ }^{30}$ R. B. Thompson and E. F. Lopes, "The effects of focusing and refraction on Gaussian ultrasonic beams," J. Nondestruct. Eval. 4(2), 107-123 (1984).

${ }^{31}$ R. L. Weaver, "Diffusivity of ultrasound in polycrystals," J. Mech. Phys. Solids 38(1), 55-86 (1990).

${ }^{32}$ P. H. Rogers and A. L. Van Buren, "An exact expression for the Lommeldiffraction correction integral," J. Acoust. Soc. Am. 55(4), 724-728 (1974). 
${ }^{33}$ Information on the attenuation of ethylene glycol available at http:// www.kayelaby.npl.co.uk/general_physics/2_4/2_4_1.html (Last viewed April 8, 2019).

${ }^{34} \mathrm{~S}$. Ahmed and R. B. Thompson, "Attenuation of ultrasonic waves in cubic metals having elongated, oriented grains," Nondestruct. Test. Eval. 8-9(1-6), 525-531 (1992).

${ }^{35}$ A. P. Arguelles and J. A. Turner, "Ultrasonic attenuation of polycrystalline materials with a distribution of grain sizes," J. Acoust. Soc. Am. 141(6), 4347-4353 (2017).
${ }^{36}$ A. G. Every and A. K. McCurdy, "Second and higher order elastic constants," in Landolt-Börnstein Numerical Data and Functional Relationships in Science and Technology New Series Group III, edited by D. F. Nelson (Springer-Verlag, Berlin, 1992), Vol. III/29a, p. 1.

${ }^{37}$ See supplementary material at http://dx.doi.org/10.1121/1.5139220 for the matlab code provided to calculate the elastic covariance for crystallites of any symmetry class, along with the inner products for random wave propagation and displacement directions. 